Annuaire du Collège de France 2017-2018

\title{
Civilisation pharaonique : archéologie, philologie, histoire
}

Nicolas Grimal

\section{(2) OpenEdition}

1 Journals

Édition électronique

URL : https://journals.openedition.org/annuaire-cdf/15723

DOI : $10.4000 /$ annuaire-cdf.15723

ISBN : 978-2-7226-0572-5

ISSN : 2109-9227

Éditeur

Collège de France

Édition imprimée

Date de publication : 30 décembre 2020

Pagination : 181-186

ISBN : 978-2-7226-0516-9

ISSN : 0069-5580

\section{Référence électronique}

Nicolas Grimal, « Civilisation pharaonique : archéologie, philologie, histoire », L'annuaire du Collège de

France [En ligne], 118 | 2020, mis en ligne le 01 avril 2021, consulté le 22 août 2022. URL : http://

journals.openedition.org/annuaire-cdf/15723; DOI : https://doi.org/10.4000/annuaire-cdf.15723

Tous droits réservés 


\title{
CIVILISATION PHARAONIQUE : ARCHÉOLOGIE, PHILOLOGIE, HISTOIRE
}

\author{
Nicolas GRIMAL \\ Membre de l'Institut (Académie des inscriptions et belles-lettres), \\ professeur au Collège de France
}

Mots-clés : histoire, philologie, civilisation pharaonique, littérature égyptienne, Égypte

\section{ENSEIGNEMENT}

COURS - LE CALAME ET LA PIERRE. ESSAI D'HISTOIRE CRITIQUE DE LA LITTÉRATURE ÉGYPTIENNE ANTIQUE (SUITE)

Nous avons terminé cette année l'analyse du passage du récit à la première personne au genre littéraire de l'autobiographie ${ }^{1}$ en traduisant et commentant les récits de Hirkouf et Pepynakht ${ }^{2}$ et en comparant ces récits aux realia historiques et géographiques. Le même travail a été mené, en parallèle, avec la campagne de l'an 23 de Thoutmosis III ${ }^{3}$.

Passant de l'autobiographie au récit littéraire, le premier texte étudié a été le « roman » de Sinouhé, qui a été traduit et commenté selon la même méthode, en apportant une attention particulière à l'intertextualité, tant vers les sources contemporaines, ou supposées telles, comme l'Enseignement d'Amenemhat I Ir, les

1. R. SALIM, Cultural Identity and Self-presentation in Ancient Egyptian Fictional Narratives: An Intertextual Study of Narrative Motifs from the Middle Kingdom to the Roman Period, Copenhague, 2013; A. WIEDER, Altägyptische Erzählung. Form und Funktion einer literarischen Gattung, Heidelberg, 2007 ; M.E. HorbURY, Egyptian Self-Definition in the New Kingdom and the Coptic Period, Londres, University College, 2003.

2. E. EDEL, «Inschriften des Alten Reiches. V. Die Reiseberichte des Hrw-hwwjf (Herchuf) », DAWB IfO, 1955, p. 59 sq.

3. Annales I, 9-11; 18-56. Cf. N. GRIMAL, «Civilisation pharaonique: archéologie, philologie, histoire », Annuaire du Collège de France 2002-2003. Résumé des cours et travaux, vol. 103, 2003, p. 730-731. 
papyrus de Kahun et Gurob, les Admonitions, etc. ${ }^{4}$. - qu'en direction de fonds postérieurs, comme le papyrus Anastasi II ou la stèle triomphale de Py(ânkh)y, voire hors de la culture égyptienne, comme la geste de Gilgamesh ou I Sam 17 pour le combat contre le champion du Retenou. Ce dernier épisode, comme celui de la fuite hors d'Égypte qui ouvre le récit, a également été l'occasion d'avancer de premiers éléments sur la postérité littéraire égyptienne et de marquer la différence entre citations et références culturelles.

On a ainsi, chemin faisant, isolé quelques éléments caractéristiques de la nature littéraire du roman: mélange des genres et des styles (récit à la $1^{\text {re }}$ personne, dialogues, lettres, passages hymniques, métaphores politiques, développements sous forme de documents administratifs, etc.), procédés littéraires (lexique littéraire et diglossie, prosodie ${ }^{5}$, métaphores littéraires, prosopopée, répétitions, citations, introduction de documents extérieurs, etc.). Ces contours sont communs à tout ce que l'on a qualifié de "roman du voyage ${ }^{6}$ », du franchissement des frontières au bannissement, sans oublier l'intervention divine (fatum et deus ex machina), qui font évidemment penser au roman picaresque, tel que le campent, dès l'Antiquité, des œuvres comme les Éthiopiques d'Héliodore d'Émèse.

Le deuxième exemple de « roman du voyage » étudié cette année a été le conte du Naufragé, que sa trame narrative place parmi ces récits de l'errance, mais dont la construction fait encore plus appel à des ressorts proprement littéraires ${ }^{7}$. Fondé sur une construction en abyme, il se présente sous la forme d'un apologue, construit comme élément d'un dialogue. Mais celui-ci reste fictif, le destinataire de l'apologue se contentant d'une remarque aussi désabusée que lapidaire en matière de conclusion. Le style, comme le lexique utilisé, démentent l'aspect anodin de ce conte, qui a parfois été considéré comme une simple fable et rangé sur l'étagère des récits merveilleux. À la fois sagesse et récit initiatique, il constitue l'un des rares exemples de récit moral ${ }^{8}$, ce qui lui vaut une certaine postérité littéraire 9 .

4. Cf., par exemple, J.P. AlLEN, «The historical inscription of Khnumhotep at Dahshur: Preliminary report », BASOR, vol. 352, 2008, p. 36 ; R.B. PARKINSON, Reading Ancient Egyptian Poetry: Among Other Histories, Chichester, Wiley-Blackwell, 2009.

5. J.L. Foster, Thought Couplets in "The Tale of Sinuhe": Verse Text and Translation with an Outline of Grammatical Forms and Clause Sequences and an Essay on the Tale as Literature, Francfort-sur-le Main/New York, P. Lang, 1993.

6. G. MoERS, Fingierte Welten in der ägyptischen Literatur des 2. Jahrtausends v. Chr. Grenzüberschreitung, Reisemotiv und Fiktionalität, Leyde/Boston, Brill, coll. «Probleme der Ägyptologie », vol. 19, 2001.

7. A. LOPRIENO, «The sign of literature in the Shipwrecked Sailor », in U.VERHOEVEN et E. GRAEFE (dir.), Religion und Philosophie im alten Ägypten. Festgabe für Philippe Derchain zu seinem 65. Geburtstag am 24. Juli 1991, Louvain, Departement oriëntalististiek/Peeters, coll. «OLA », vol. 39, 1991, p. 209-217; G. BURKARD, Überlegungen zur Form der ägyptischen Literatur. Die Geschichte des Schiffbrüchigen als literarisches Kunstwerk, Wiesbaden, Harrassowitz, coll. « ÄAT », vol. 20, 1993 ; G. MOERS, op. cit.

8. Cette étude a fait l'objet d'une communication, intitulée «La sagesse du naufragé ? » à l'Académie des Inscriptions \& Belles-Lettres, le 8 février 2019.

9. W.K.S. SIMPSON, JAOS, vol. 78, 1958, p. 50-51; Aménémopé, 3.8-4.2; J. ČERNÝ et A.H. GARDINER, Hieratic Ostraca, vol. 1, Oxford, Oxford University Press, 1957, p. 22, pl. 78, 79 (fac-similé), 78A, 79A (transcription) ; Stèle de la Victoire, 51-52 : N. GRIMAL, La Stèle triomphale de Pi('ânkh)y au Musée du Caire (JE 48862 et 47086-47089), Le Caire, Institut 
Restant dans le domaine du « roman du voyage », on a traduit et étudié ensuite le Rapport d'Ounamon. Connu par un seul manuscrit, découvert dans une jarre avec d'autres papyrus, par des paysans de la région de El-Hibeh, il appartenait à un lot de trois textes différents, qui furent l'objet d'un partage « sauvage » entre les inventeurs. Le Rapport fut acheté en 1891 au Caire par Wladimir Golenischeff. Les trois œuvres sont aujourd'hui conservées au musée Pushkin de Moscou. Ce sont les pMoscou 120 (Ounamon), 127 (la «Lettre littéraire de Moscou », connue également sous le nom des Mésaventures d'Ourmaï), et 169 (l'onomasticon d'Aménopé) ${ }^{10}$. Cette proximité d'œuvres ancrées dans la géographie et l'espace suffirait, à elle seule, à justifier la définition de «roman du voyage». Elle est surtout révélatrice de la culture du propriétaire de cette « bibliothèque ».

\section{SÉminAIRE - Les ANNALES DE THOUTMOSIS III (SUITE ET FIN)}

Nous avons étudié cette année les dernières colonnes de la section VII des Annales (VII, col. 21-33), soit la fin et la conclusion des dotations au temple conservées sur la face orientale du môle sud du VII ${ }^{\mathrm{e}}$ pylône :

(21) $[n$ mh.n=i $h r] w d t .=f i r t$ $m$ ht $3 b . n=f$ hpr

$m \operatorname{mrt} n b(t) k 3=f$

$i r=i n=f$ st $m i$ wd $=f$

$h 3 t y=i h r h r p(w) i$

"wy=i hr irt $n$ it $t=i \mathrm{~km} 3$ wi

hr irt 3ht nbt $n$ it $=i$

(22) [ist i] $n$ hm=i gm 3ht nbt

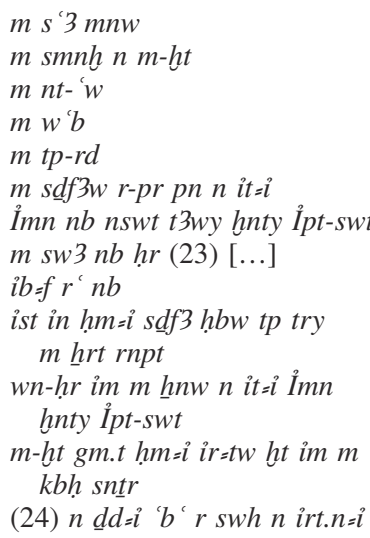

«(21) [je n’ai pas négligé ce] qu'il a ordonné de faire de ce qu'il voulait voir exister,

de tout ce que souhaite son $k a$.

Je le fais pour lui comme il l'a ordonné,

guidé par mon cœur.

Mes mains d'agir pour mon père qui m’a élevé, faisant toutes œuvres utiles à mon père.

(22) C'est bien Ma Majesté qui trouve tout ce qui est utile :

agrandir les monuments,

(les) remettre en état pour le futur,

rites,

purification,

règlements,

approvisionnement de ce temple de mon père

Amon, Seigneur des trônes du Double Pays, qui réside

à Ipet-sout, à chaque passage (23) [...]

son cœur chaque jour.

C'est bien Ma Majesté qui alimente les fêtes saisonnières durant l'année,

attentif ici dans le palais de mon père Amon qui est à la tête d'Ipet-sout,

après que Ma Majesté ait constaté que l'on accomplissait le rite ici avec libation et encens.

(24) Je ne dis pas de vantardise pour qu'on chante les louanges de ce que j'ai fait,

français d'archéologie orientale, coll. «Études sur la propagande royale égyptienne », vol. 1, 1981 , p. $284,20^{*}$.

10. B.U. SCHIPPER, Die Erzählung des Wenamun: ein Literaturwerk im Spannungsfeld von Politik, Geschichte und Religion, Fribourg/Göttingen, Academic Press/Vandenhoeck \& Ruprecht, coll. « OBO », vol. 209, 2005, p. 3 sq. 
$r \underline{d} d$ ir.nỉ hnw

iw $n$ ir $s w h m=i$

nn ir.n=i $n$ rmtit

$d d=t w$ ' $b$ ' $r=s$

$i r . n=i$ nn $n$ it $=i$ (25)

$[n$ hm. $n=f p 3] d d$ hn nty

$n$ ir.tw $=f$

$r-n t t$ sw rh pt rh t3

$m 3=f t 3 r-\underline{d} r=f n w n w t$

'nh $n=i$ mry wi $R$

hs wi it $=i \stackrel{I}{I} m n$

hwn fnd $=i m$ ' $n h$ w3s

iw ir.n=i $n n$ (26) [...]

w'bw twri=tn hr iht-ntrr

s3w tn hr sp n wnt

$s 3 k[?] w i b=t n$

tmm $r 3=\underline{t} n h r m$ 3. $s$ nb $r \quad r d=f$

imi (27) $[\ldots]$

(27) [imi hknw] $n$ twwt $=i$

$r-d 3 w t$ mnw ir. $n=i$

$s^{\prime} r \underline{t n} n=i$ prt $m-b 3 h$ mi $s h b=i$ $r 3-p r=f$

db3 mnht twwt =i m sšr mi $m h=i$ prw-hd $m p 3 k t$

(28) [...] hknw nsi $m$ rnpwt

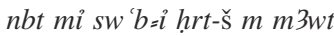
$d i=\underline{i} n n=i$ 3šrt stpt mi hitr $=i$ tp-trw $m$ iw $3 w$

$m h w n=i \quad h 3 w$ irtt

$i w \operatorname{snt} r[\ldots]$

(29) $[\ldots]$ wdh $m$ h $\underline{d} n$ nwb

$k^{\prime} h{ }^{\prime}=\underline{t} n n$ twwt $=i$ hft snmt $n j$ imyw-b3h

$s \underline{t} 3$ twt $=i$ hrw hnt $w y=\underline{t} n \underline{h r}$ rdit hknw

i it $t=i$ ip $=$ s st $r-\underline{d} 3 w t$ ir.t.n=i $m[\ldots]$

(30) $w 3[h] \cdot n[n=f] h m[=i]$ $h t p w-[n t r] m m 3 w t$

$m$ hrt-hrw nt $r^{\prime} n b \quad m-h 3 w$ $w n(n) t m-b 3 h$

$m t$ šbnw $n$ htp-ntr 3305

hnkt ds 133

$d k w t h \underline{d} 2$

$3 h n d 32$

bnr $n d 32$

ht ' 3 šd 2

(31) $[i w$ w $3 h . n n=f h m=i$ pour que l'on dise que j'ai accompli une épopée,

alors que Ma Majesté ne l'a pas fait.

Je n'ai pas agi pour le peuple,

pour qu'on le qualifie de vantardise!

Je l'ai fait pour mon père (25) [Amon...]

[Il n'ignore personne] qui raconte une épopée, qu'il n'a pas accomplie.

Car il connaît le ciel, il connaît la terre

et voit la terre tout entière en un instant.

Aussi vrai que Rê vit pour moi et m'aime, que me loue mon père Amon,

que mon nez fleurit de vie et de force,

j'ai fait cela (26) [...]

Prêtres ouab, soyez attentifs à vos tâches.

Ne vous relâchez dans aucun de vos rituels.

Soyez purs pour accomplir le service divin.

Gardez-vous de vous laisser aller à la négligence.

Maîtrisez vos cœurs.

Gardez votre bouche close sur ce que chacun a vu pendant son service.

Faites que (27) [...]

(27) [Donnez des louanges] à mes statues en échange du monument que j'ai érigé.

Élevez pour moi l'offrande qui a été présentée, puisque j'ai organisé les fêtes de son temple.

Mettez un vêtement de lin à mes statues, puisque j'ai rempli les magasins de lin fin.

(28) $[\ldots]$ des offrandes pour moi consistant en toutes sortes de plantes fraîches,

puisque j'ai purifié à nouveau le $h r t$-šs.

Puissiez-vous me donner des morceaux de choix, puisque j'ai fixé pour les fêtes de début de saisons une dotation en bœufs.

Remplissez pour moi une coupe de lait,

tandis que l'encens $[\ldots]$

(29) $[\ldots]$ un autel en argent et or.

Pliez votre bras pour mes statues après avoir nourri les ancêtres.

Tirez ma statue le jour de la procession, les bras levés pour me louer.

Eh ! c'est que mon père paye à proportion de ce que j'ai fait de [...]

(30) [Ma] Majesté [lui] a consacré à nouveau des offrandes [divines] pour chaque jour en plus de ce

qu'il y avait avant,

consistant en 3305 différentes sortes de pains

d'offrande divine,

133 mesures $d s$ de bière,

2 pains de fine farine $d k w$,

2 blocs de pâte $3 h$,

2 blocs de dattes,

2 oies grand bois grasses.

(31) Et Ma Majesté a consacré pour lui une offrande 


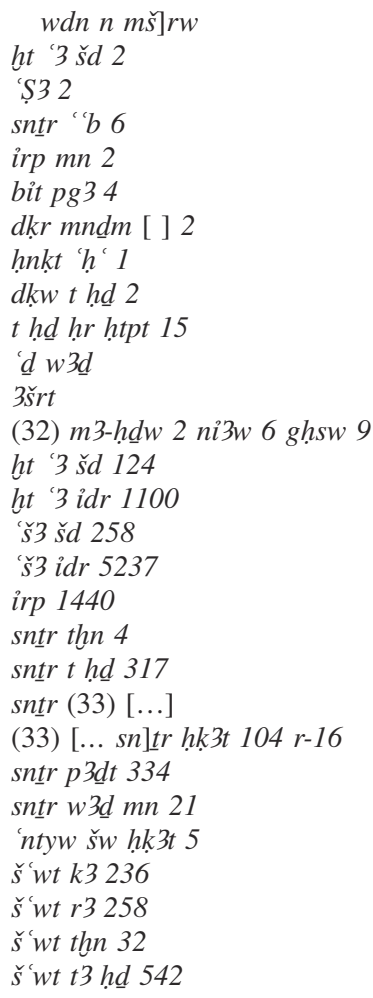

du soir :

2 oies grand bois,

2 volatiles,

6 mesures ' $b$ d'encens,

2 cruches $m n$ de vin,

4 coupes $p g 3$ de miel,

$(\mathrm{x}+) 2$ corbeilles de fruits,

1 vase ' $h$ de bière,

2 pains blancs de farine $d k w$,

15 pains blancs sur la table d'offrande,

de la graisse fraîche,

des pièces de viande,

(32) 2 gazelles, 6 oryx, 9 ibex,

124 oies grand bois engraissées,

1100 oies grand bois domestiquées,

258 volatiles engraissés,

5237 volatiles domestiqués,

1440 cruches de vin,

4 encens en forme d'obélisques,

317 encens en forme de pain blanc, encens (33) $[\ldots]$

(33) $[\ldots]$ [En]cens 104 1/16 mesures heqat, encens $334 p \underline{d} t$,

encens frais 21 mesures $m n$,

myrrhe sèche 5 heqat,

236 gâteaux en forme de taureau,

258 gâteaux en forme d'oie,

32 gâteaux en forme d'obélisque,

542 gâteaux en forme de pain blanc. »

\section{TRAVAUX \& PUBLICATIONS}

\section{CONFÉRENCES, COLLOQUES ET COMMUNICATIONS}

Les contraintes éditoriales de l'Annuaire du Collège de France ne permettant pas d'inclure dans ce compte rendu l'ensemble des activités de l'équipe et de la bibliothèque du Cabinet d'égyptologie, on trouvera la version exhaustive de ce rapport sur le site internet de la chaire (http://www.egyptologues.net/chaire/rapports/ rapports.htm).

\section{Publications}

AdLy E. et GRImAL N., «Un grand v(b)ide qui sonne le creux », Bulletin d'information archéologique, vol. 56, 2017.

ADLY E. et GRIMAL N., «Les Fatimides au Canada », Bulletin d'information archéologique, vol. 57, 2018.

Francis-Allouche M., Grimal N., Abdul Massih H., Herbich T., Carayon N., Papatheodorou G., Geraga M., Georgiou N., Christodoulou D., Mathé V. et ADRIEN C., «Byblos maritime : une installation portuaire au piémont sud de la ville antique », $B A A L$, vol. 17, 2017, p. 171-234. 
Gaber Kerious H., Grimal N. et Perdu O. (dir.), Imitations, copies et faux dans les domaines pharaonique et de l'Orient ancien. Actes du colloque Collège de France-Académie des Inscriptions et Belles-Lettres, Paris, 14-15 janvier 2016, Paris, Aibl/Soleb, coll. «Études d'égyptologie », vol. 16, 2018.

GRIMAL N., «Le dispositif archéologique français à l'étranger », Mondes et Cultures, vol. 77, $\mathrm{n}^{\text {os }} 1-4,2017$, p. 277-281, HAL : hal-01679499.

Grimal N., «Éloge de Jean-Pierre le Gall», Mondes et Cultures, vol. 77, $\mathrm{n}^{\text {os }} 1-4,2017$, p. 144-149.

GRIMAL N., Civilisation pharaonique : archéologie, philologie, histoire. Rapport d'activités 2016-2017, Paris, Collège de France, 2017, p. 1-21.

GRIMAL N., «Civilisation pharaonique: archéologie, philologie, histoire », Annuaire du Collège de France 2015-2016. Résumé des cours et travaux, vol. 116, 2018, p. 211-227, DOI : 10.4000/annuaire-cdf.12844; en ligne : https://journals.openedition.org/annuaire-cdf/12844.

Grimal N., «Du vrai et du faux », in H. GABER Kerious, N. Grimal et O. PERDu (dir.), Imitations, copies et faux dans les domaines pharaonique et de l'Orient ancien. Actes du colloque Collège de France -Académie des Inscriptions et Belles-Lettres, Paris, 14-15 janvier 2016, Paris, Aibl/Soleb, coll. « Études d'égyptologie », vol. 16, 2018, p. 6-17.

Grimal N., Hommages de M. AbD El-RazIQ, G. CASTEl et P. TAllet, Ayn Soukhna, vol. III : Le Complexe de galeries-magasins. Rapport archéologique (Le Caire, coll. «Fouilles de l'Institut français d'archéologie orientale », vol. 74, 2016) et de V. FRANCIGNY, Les Coutumes funéraires dans le royaume de Méroé. Les enterrements privés (Paris, Éditions de Boccard, coll. «Orient \& Méditerranée. Archéologie», vol. 22, 2016), CRAIBL 2017/II, 2018, p. 783-787.

GRIMAL N., Hommages de V. MATOÏAN et M. AL-MAQDISSI (dir.), Études ougaritiques, vol. III (Louvain, Peeters, coll. "Ras Shamra-Ougarit, vol. 21, 2013), de V. MATOÏAN et M. AL-MAQDISSI (dir.), Études ougaritiques, vol. IV (Louvain, Peeters, coll. « Ras ShamraOugarit, vol. 24, 2016) et de B. GEYER, V. MATOÏAN et M. AL-MAQDisSI (dir.), De l'île d'Aphrodite au Paradis perdu, itinéraire d'un gentilhomme lyonnais, en hommage à Yves Calvet (Louvain, Peeters, coll. « Ras Shamra-Ougarit », vol. 22, 2015), CRAIBL 2017, 2018, p. 580-587.

Grimal N., Hommage de M. MaILlot, Palais et grandes demeures du royaume de Méroé (Paris, PUPS, 2016), CRAI 2017, 2018, p. 266-268.

BAudelot C., Gadmer P., GiRonimi R., GRimal N., Le Quellec J.-L., LOUYS V., Richer I. et RoBert C., L'École, laboratoire de la fraternité. Contribution de L'Anthropologie pour tous sur les bénéfices scolaires de l'enquête, Aubervilliers, Traces: L'Anthropologie pour tous, coll. « OLO », vol. 4, 2017 ; en ligne : https://docs.wixstatic.com/ugd/323914_47714a54 2ce0465db45d8706e831a5e5.pdf.

Baudelot C., Grimal N., Le Quellec J.-L., Louys V., Richer I. et RoberT C., À l'école de l'enquête. Actes du troisième colloque de l'Anthropologie pour tous, Aubervilliers, Traces/ L'Anthropologie pour tous, coll. «OLO », vol. 3, 2017 ; en ligne : https://docs.wixstatic.com/ ugd/323914_c73ee7cecde747b8b16ffb4ecfb7b487.pdf. 